Sharif University of Technology
Scientia Iranica
SCIENTIA
I RAN I C A
http://scientiairanica.sharif.edu

Invited Paper

\title{
A computational plastic-damage method for modeling the FRP strengthening of concrete arches
}

\author{
T. Ahmadpour ${ }^{\mathrm{a}, \mathrm{b}}$, Y. Navid Tehrani ${ }^{\mathrm{a}}$, and A.R. Khoei ${ }^{\mathrm{a}, *}$ \\ a. Center of Excellence in Structures and Earthquake Engineering, Department of Civil Engineering, Sharif University of \\ Technology, Tehran, P.O. Box 11155-9313, Iran. \\ b. Department of Civil Engineering, School of Science and Engineering, Sharif University of Technology, International Campus, \\ Kish Island, P.O. Box 76417-76655, Iran.
}

Received 1 February 2019; accepted 11 March 2019

\author{
KEYWORDS \\ Concrete arches; \\ FRP retrofitting; \\ Reinforced concrete; \\ Plastic-damage model; \\ Cohesive fracture \\ model.
}

\begin{abstract}
In this paper, a computational technique is presented based on a concrete plastic-damage model to investigate the effect of FRP strengthening of reinforced concrete arches. A plastic-damage model was utilized to capture the behavior of concrete. The interface between the FRP and concrete was modeled using a cohesive fracture model. In order to validate the accuracy of the damage-plastic model, a single element was employed under monotonic tension, monotonic compression, and cyclic tension loads. An excellent agreement was observed between the predefined strain-stress curve and that obtained by the numerical model. Furthermore, the accuracy of the cohesive fracture model was investigated by comparing the numerical results with those of experimental data. Finally, in order to verify the accuracy of the proposed computational algorithm, the results were compared with the experimental data obtained through two tests conducted on reinforced concrete arches strengthened with FRP.

(C) 2019 Sharif University of Technology. All rights reserved.
\end{abstract}

\section{Introduction}

FRP strengthening of concrete is one the most effective retrofitting methods. In fact, a remarkable development has taken place in retrofitting with the introduction of FRP materials. The unique features of FRP include high durability against environmental degradation factors, high tensile strength, simple implementation, low weight, and easy transportation. Hence, a great deal of work has been dedicated to investigating the structural retrofit using the FRP materials in the last two decades. Most of the studies have been

\footnotetext{
*. Corresponding author. Tel.: +98 (21) 6600 5818; Fax: +98 (21) 66014828

E-mail address: arkhoei@sharif.edu (A.R. Khoei)
}

focused on the beams, columns, flat slabs, and masonry structures, while few studies have been performed on the behavior of FRP strengthening of concrete arches. Since the arch members are utilized in many structures such as bridges, fluid storage tanks, tunnels, and domes, study of the methods for retrofitting these structures has gained interest, recently.

There are several studies performed on the masonry arches retrofitted with FRP materials that present a significant improvement in structural performance $[1,2]$. The effect of FRP strengthening of concrete arch structures was studied by Chen et al. [3]. They experimentally investigated the response of concrete arch structures retrofitted by wrapping FRP carbon sheets subjected to explosive impulses. Hamed et al. [4] studied the performance of a refined concrete arch retrofitted with externally bonded composite materials, and observed that the maximum load 
increased by about 40\%. Dagher et al. [5] studied the bending behavior of concrete-filled tubular FRP arches in bridge structures. To improve the strength, stiffness, and ductility of reinforced concrete arches, Zhang et al. [6] performed a set of experimental tests to study the behavior of reinforced concrete arches retrofitted with FRP materials.

Characteristics of the concrete plastic behavior cannot generally be described using the classical theory of plasticity. Many attempts have been made to demonstrate the behavior of concrete with the classical theory of plasticity. Feenstra and de Borst [7] proposed two yield criteria to describe the concrete behavior in tension and compression that showed great agreement with experimental data in the biaxial monotonic loading condition. Cervenka and Papanikolaou [8] presented the three-dimensional combined fracture-plastic model for concrete, in which the crack band model was used together with the smeared crack model to capture the fracture in tension. Moreover, the behavior of concrete in compression was captured by the MenetreyWilliam failure model, which was capable of simulating crushing under high confinement, cracks in concrete, and closure due to crushing in different directions. Recently, a three-invariant cap plasticity model was developed by Khoei and Azami [9] and DorMohammadi and Khoei [10] to describe the plastic deformation of granular materials involving the isotropic-kinematic hardening and associated plasticity flow rule.

Basically, damage happens in the concrete due to micro-cracks; in fact, micro-cracks occur because of thermal expansion at the interface between the cement and aggregate. Several studies have been carried out to model the damage in concrete structures. Bažant and Ožbolt [11] proposed a nonlocal micro-plane model for the fracture, damage, and size effect in concrete structures. Voyiadjis and Abu-Lebdeh [12] presented a damage model based on the bounding surface concept for concrete behavior. The combined plastic-damage model has been used by researchers to capture the stiffness degradation of concrete, in which the damage variables are assumed according to the plastic deformation in a constitutive formulation to calibrate parameters with experimental data. In the plastic-damage model, the irreversible plastic phenomenon can be modeled using the concept of plasticity in the effective stress space, while the stiffness degradation can be captured using the continuum damage mechanics. Lubliner et al. [13] and Yazdani and Schreyer [14] presented a combined plastic-damage mechanics model for plain concrete. Kattan and Voyiadjis [15,16] proposed a coupled theory of damage mechanics and finite strain elasto-plasticity. Lee and Fenves [17] described a plastic-damage model for concrete based on the concept of fracture-energy and stiffness degradation under cyclic loading. Faria et al. [18] presented a strain-based plastic viscous- damage model for massive concrete structures. Salari et al. [19] proposed a triaxial constitutive model for elastoplastic behavior of geomaterials that captured tensile damage. Since the behavior of concrete is different in tension and compression, different damage models are required for each type of loading; however, some studies consider only one damage variable for different loading conditions [20,21]. On the other hand, it is important to model the concrete damage using two separate damage variables for tension and compression $[22,23]$. The plastic-damage models have been applied based on different damage variables for tension and compression for both brittle and ductile materials [24-27].

Considering the beneficial characteristics of FRP materials, as well as the ease of implementation of these materials in architectural elements, an investigation into the behavior of reinforced concrete arch structures strengthened with FRP materials is worthwhile. To this end, both the experimental and numerical investigations are required to obtain the behavior of FRP strengthening in concrete arch structures. In this study, a computational technique is presented based on the plastic-damage model to investigate the effect of FRP strengthening on concrete arches. A plasticdamage model is utilized to capture the behavior of concrete. The interface between the FRP and concrete is modeled using a cohesive fracture model. To validate the accuracy of the concrete model, a single element is considered and subjected to monotonic tension, monotonic compression, and cyclic tension loads. Good agreement is observed between the predefined strainstress curve and the strain-stress curve obtained by the numerical analysis. Furthermore, the accuracy of the interface model is investigated by comparing the results with the experimental data. In order to verify the computational model of the arch, the experimental data of two tests conducted on concrete arches strengthened with FRP are utilized.

\section{Plastic-damage model for concrete}

In this study, the plastic-damage model originally proposed by Lubliner et al. [13] and Lee and Fenves [17] and then, employed by Nguyen et al. [21,22] is employed, in which the plastic-damage model is developed on the basis of the thermo-dynamical approach. The strain tensor $\varepsilon$ is decomposed into the elastic part $\varepsilon^{e}$ and the plastic part $\varepsilon^{p}$, in which the relation between stress and strain is defined as:

$$
\sigma=E:\left(\varepsilon-\varepsilon^{p}\right) \text {, }
$$

where $E$ is elasticity modulus, $\sigma$ is stress, and $\varepsilon$ and $\varepsilon^{p}$ are total strain and plastic strain, respectively. By mapping the stress onto the effective stress space, the plasticity and damage equations can be solved 
independently. Applying the scalar damage variable $D$, the effective stress can be expressed as:

$$
\bar{\sigma}=(1-D) E_{0}:\left(\varepsilon-\varepsilon^{p}\right),
$$

where $E_{0}$ is the undamaged elastic-stiffness modulus. Considering the non-associated flow rule, the strain rate $\dot{\varepsilon}^{p}$ can be defined as:

$$
\dot{\varepsilon}^{p}=\dot{\lambda} \nabla_{\bar{\sigma}} \Phi(\bar{\sigma})
$$

where $\dot{\lambda}$ denotes the plastic consistency parameter, which is a non-negative function, and $\Phi$ is a scalar plastic potential function. Moreover, the damage variable $k$ is required to represent the damage states variable as:

$$
\dot{k}=\dot{\lambda} H(\bar{\sigma}, k) \text {. }
$$

\subsection{The damage model}

In order to represent the tensile and compressive damages to concrete material, two damage variables are defined. These damage variables have values between zero and one for covering the range from undamaged to completely damaged concrete. In the Barcelona model introduced by Lubliner et al. [13], the uniaxial stress is defined as a function of the plastic strain, i.e.:

$$
\sigma=f_{0}\left[(1+a) \exp \left(-b \varepsilon^{p}\right)-a\left(\exp \left(-2 \varepsilon^{p}\right)\right)\right],
$$

where $a$ and $b$ are dimensionless constants and $f_{0}$ is the initial yield stress. Consider an exponential form for the degradation $D$ as:

$$
1-D=\exp \left(-c \varepsilon^{p}\right)
$$

The effective stress can be written as:

$$
\bar{\sigma}=f_{0}\left[(1+a)\left(\exp \left(-b \varepsilon^{p}\right)\right)^{1-\frac{c}{b}}-a\left(\exp \left(-b \varepsilon^{p}\right)\right)^{2-\frac{c}{b}}\right],
$$

in which the damage variable for the uniaxial loading is denoted by $k$, defined as:

$$
k=\frac{1}{g} \int_{0}^{\varepsilon^{p}} \sigma\left(\varepsilon^{p}\right) d \varepsilon^{p}
$$

where $g$ is defined as:

$$
g=\int_{0}^{\infty} \sigma\left(\varepsilon^{p}\right) d \varepsilon^{p}
$$

in which the quantity is the dissipated energy density during the forming of microcracking [17]. Substituting Eq. (5) into Eq. (9), the relation between $g, a$, and $b$ can be defined as:

$$
g=\frac{f_{0}}{b}\left(1+\frac{a}{2}\right) \text {. }
$$

The uniaxial stress can be defined in terms of $k$ by combining Eqs. (8) and (10) as:

$$
\sigma=\frac{f_{0}}{a}[(1+a) \sqrt{1+a(2+a) k}-1+a(2+a) k] .
$$

Moreover, the effective stress can be expressed in terms of damage variable as:

$$
\bar{\sigma}=\frac{f_{0}}{a}[(1+a)-\sqrt{1+a(2+a) k}]^{1-\frac{c}{b}}(\sqrt{1+a(2+a) k}) \text {. }
$$

Hence, $D$ can be defined as:

$$
D=1-[(1+a)-\sqrt{1+a(2+a) k}]^{\frac{c}{b}} .
$$

By taking derivative from Eq. (8), the damage evolution equation for the uniaxial state can be written as:

$$
\dot{k}=\frac{1}{g} f(k) \dot{\varepsilon}^{p} .
$$

To convert damages from a uniaxial damage evolution to a multi-axial damage evolution, plastic strain rate is calculated by the following equation:

$$
\dot{\varepsilon}^{p}=\delta_{t} r(\bar{\sigma}) \dot{\varepsilon}_{\max }^{p}+\delta_{c}(1-r(\bar{\sigma})) \dot{\varepsilon}_{\min }^{p},
$$

where $\delta$ is the Kronecker delta, $\dot{\varepsilon}_{\min }^{p}$ and $\dot{\varepsilon}_{\max }^{p}$ are algebraically the minimum and maximum eigenvalues of the plastic strain rate tensor, and $r(\bar{\sigma})$ is a weight factor expressed as:

$$
r(\bar{\sigma})= \begin{cases}0, & \text { if } \bar{\sigma}=0 \\ \left(\sum_{i=1}^{3}\left\langle\bar{\sigma}_{i}\right\rangle\right) /\left(\sum_{i=1}^{3}\left|\bar{\sigma}_{i}\right|\right), & \text { otherwise }\end{cases}
$$

Substituting Eq. (12) into Eq. (1), the evolution equation can be obtained:

$$
\dot{k}=h(\bar{\sigma}, k): \dot{\varepsilon}^{p},
$$

where:

$$
h(\bar{\sigma}, k)=\left[\begin{array}{ccc}
r(\bar{\sigma}) f_{t}\left(k_{t}\right) / g_{t} & 0 & 0 \\
0 & 0 & (1-r(\bar{\sigma})) f_{c}\left(k_{c}\right) / g_{c}
\end{array} \text { i18 }_{(18)}\right.
$$

Finally, applying the definition of $\dot{\varepsilon}^{p}$ to Eq. $(3), H(\bar{\sigma}, k)$ can be obtained as:

$$
H=h \cdot \nabla_{\bar{\sigma}} \Phi(\bar{\sigma}) .
$$

\subsection{The plasticity model and hardening functions}

Yield criterion is the most important part of the plasticity model. It is employed to model the behavior of concrete under the tensile and compressive loadings. Implementation of similar behavior of tension and compression in concrete leads to an unrealistic plastic deformation [13]. In this study, the yield criterion originally introduced by Lubliner et al. [13] and then, modified by Lee and Fenves [17] is proposed. The yield 
function is defined in the effective stress space using the undamaged configuration parameters as:

$$
F(\sigma)=\frac{1}{1-\alpha}\left[\alpha I_{1}+\sqrt{3 J_{2}}+\beta \sigma_{\max }\right]-c_{c}(k),
$$

where $I_{1}$ is the first invariant and $J_{2}$ is the second invariant of the effective deviatoric stress tensor. In the above equation, $\alpha$ and $\beta$ are dimensionless constants, in which $\alpha$ depends on the ratio of yield strength under the biaxial and uniaxial compressions, defined as [13]:

$$
\alpha=\frac{\left(f_{b 0} / f_{c 0}\right)-1}{2\left(f_{b 0} / f_{c 0}\right)-1},
$$

where $f_{b 0}$ is the biaxial and $f_{c 0}$ is the uniaxial compressive yield stress. The experimental values of $f_{b 0} / f_{c 0}$ are within the range of 1.10 and 1.16, which leads the value of $\alpha$ to be between 0.08 and 0.12 . The parameter $\beta$ is a constant value that is defined as a dimensionless function of the tensile and compressive cohesion parameters $c_{t}$ and $c_{c}$ in the Barcelona model as:

$$
\beta=\frac{c_{c}(k)}{c_{t}(k)}(\alpha-1)-(1+\alpha) .
$$

The biaxial tensile strength depends on the parameters $\alpha$ and $\beta$, and is always slightly lower than the uniaxial tensile strength.

The flow rule is defined based on the relation between the plastic flow direction and the plastic strain rate. In this study, a non-associative flow rule is required to control the dilatancy in modeling the frictional behavior of material. Hence, a plastic potential function, which is of the Drucker-Prager yield criteria type, is utilized as:

$$
\varphi=\sqrt{2 J_{2}}+\alpha_{p} I_{1},
$$

where the parameter $\alpha_{p}$ is chosen such that a proper value of dilatancy is obtained.

\section{Cohesive fracture model for FRP interface}

In the cohesive fracture model, it is assumed that the fracture process zone extends along the crack faces, while in the linear elastic fracture model, the fracture occurs in the crack tip region. The basic assumption of this model is that while the fracture process zone occurs, the material is still capable of transferring stresses. The micro-cracks appear close to the interface and the macro-cracks occur by the assemblage of microcracks [28]. The simplest constitutive relation describing the fracture process zone is based on the cohesive fracture traction, which is defined as a function of the separation of two faces along the crack interface. The cohesive constitutive relation includes the tensile strength of the material $f_{t}$ and the fracture energy $E_{f}$. In this model, the effective traction and the effective crack separation are defined as:

$$
\begin{aligned}
& t^{e}=\sqrt{\left(t^{n}\right)^{2}+\left(t^{s}\right)^{2}}, \\
& \Delta^{e}=\sqrt{\left(\Delta^{n}\right)^{2}+\left(\Delta^{s}\right)^{2}},
\end{aligned}
$$

where $t^{n}$ and $t^{s}$ are the normal and tangential tractions, and $\Delta^{n}$ and $\Delta^{s}$ are the normal and sliding displacements of the fracture surface, respectively. The damage initiation occurs when the traction or separation reaches the critical value, i.e., $t^{e} \geq t^{0}$ or $\Delta^{e} \geq \Delta^{0}$.

The constitutive law can be defined between the cohesive traction $t_{j}$ and crack separation $\Delta_{i}$ using $t_{j}=t\left(\Delta_{i}\right)$ or $\dot{t}_{j}=T_{j i} \dot{\Delta}_{i}$, where $T_{j i}$ is the constitutive tangent stiffness tensor of cohesive fracture $[29,30]$. In this study, the delamination model proposed by Turon et al. [31] is utilized based on the continuum damage model. The free energy can be defined per unit area of the crack interface as:

$$
\begin{aligned}
& \varphi(\Delta, d)=(1-d) \varphi^{0}\left(\Delta_{i}\right)-d \varphi^{0}\left(\delta_{1 i}\left\langle-\Delta_{1}\right\rangle\right), \\
& \quad i=1,2,
\end{aligned}
$$

where $d$ is a scalar damage variable and $\varphi^{0}$ is a function of the displacement separation, defined as $\varphi^{0}(\Delta)=\frac{1}{2} \Delta_{i} T_{i j}^{0} \Delta_{j}$, with $T^{0}$ denoting the constitutive undamaged tangent stiffness tensor. In Eq. (26), $\langle\cdot\rangle$ is the MacAuley bracket defined as $\langle\Xi\rangle=\frac{1}{2}(\Xi+|\Xi|)$ and $\delta_{i j}$ is the Kronecker delta. The negative value of $\Delta_{1}$ means that the interface is in contact and the damage cannot occur in the normal direction. By taking the derivative from the free energy Eq. (26), the constitutive equation for the crack interface can be obtained as:

$$
t_{i}=\frac{\partial \varphi}{\partial \Delta_{i}}=(1-d) T_{i j}^{0} \Delta_{j}-d T_{i j}^{0} \delta_{1 i}\left\langle-\Delta_{1}\right\rangle .
$$

\subsection{The damage criterion and damage evolution law}

The damage criterion can be defined as a function of the displacement separation as:

$$
\bar{F}\left(\Delta^{e}, \bar{d}\right)=G\left(\Delta^{e}\right)-G(\bar{d}) \leq 0,
$$

where $\bar{d}$ is the damage threshold and $G$ is a function of damage evolution, which is defined in the range of $[0,1]$ as:

$$
G\left(\Delta^{e}\right)=\frac{\Delta^{f}\left(\Delta^{e}-\Delta^{0}\right)}{\Delta^{e}\left(\Delta^{f}-\Delta^{0}\right)} .
$$

Depending on the definition of damage, the damage evolution can be given by $d=1-T / T^{0}$. If the linear cohesive constitutive relation is considered, $T$ and $T^{0}$ can be defined as:

$$
\begin{aligned}
& T^{0}=\frac{t^{0}}{\Delta^{0}}, \\
& T=\frac{t^{e}}{\Delta^{e}}=\frac{-t^{0}\left(\Delta^{e}-\Delta^{f}\right)}{\Delta^{e}\left(\Delta^{f}-\Delta^{0}\right)} .
\end{aligned}
$$

The damage is initiated when the effective separation 
$\Delta^{e}$ exceeds the initial damage threshold. Now, it is required to define an evolution law for the damage model. The evolution law can be defined as:

$$
\dot{d}=\dot{\mu} \frac{\partial \bar{F}\left(\Delta^{e}, \bar{d}\right)}{\partial \Delta^{e}}=\dot{\mu} \frac{\partial G\left(\Delta^{e}\right)}{\partial \Delta^{e}}
$$

where $\dot{\mu}$ is the damage consistency parameter that should satisfy the loading-unloading conditions, i.e.:

$$
\dot{\mu} \geq 0, \quad \bar{F}\left(\Delta^{e}, \bar{d}\right) \leq 0, \quad \dot{\mu} F\left(\Delta^{e}, \bar{d}\right)=0 .
$$

In order to derive the constitutive tangent stiffness tensor, the damage model is implemented through a nonlinear approach. Taking the derivative from Eq. (27) leads to:

$$
\begin{aligned}
\dot{t}_{i}= & \delta_{i j} T_{i j}^{0}\left[1-d\left(1+\delta_{1 j} \frac{\left\langle-\Delta_{j}\right\rangle}{\Delta_{j}}\right)\right] \dot{\Delta}_{j} \\
& -\delta_{i j} T_{i j}^{0}\left[1+\delta_{1 j} \frac{\left\langle-\Delta_{j}\right\rangle}{\Delta_{j}}\right] \Delta_{j} \dot{d},
\end{aligned}
$$

and the evolution of the damage variable $d$ can be obtained as:

$$
\dot{d}=\left\{\begin{array}{c}
\dot{G}\left(\Delta^{e}\right)=\frac{\partial G\left(\Delta^{e}\right)}{\partial \Delta^{e}} \dot{\Delta}^{e}=\frac{\Delta^{f} \Delta^{0}}{\Delta^{f}-\Delta^{0}} \frac{1}{\left(\Delta^{e}\right)^{2}}, \\
\text { for } \bar{d}<\Delta^{e}<\Delta^{f} \\
0, \quad \text { for } \bar{d}>\Delta^{e} \text { or } \Delta^{e}>\Delta^{f}
\end{array}\right.
$$

Finally, the constitutive tangent stiffness tensor, $T_{j i}^{\mathrm{tan}}$, in the constitutive law, $\dot{t}_{j}=T_{j i}^{\mathrm{tan}} \dot{\Delta}_{i}$, can be defined as:

$T_{j i}^{\mathrm{tan}}=\left\{\begin{array}{rr}\delta_{i j} T_{i j}^{0}\left[1-d H_{j}\right]- & T_{i j}^{0} H_{j} H_{i} \frac{\Delta^{f} \Delta^{0}}{\Delta^{f}-\Delta^{0}} \frac{1}{\left(\Delta^{e}\right)^{2}} \\ & \text { for } \bar{d}<\Delta^{e}<\Delta^{f} \\ \delta_{i j} T_{i j}^{0}\left[1-d H_{j}\right], & \text { for } \bar{d}>\Delta^{e} \text { or } \Delta^{e}>\Delta^{f}\end{array}\right.$

where $H_{j}=1+\delta_{1 j}\left\langle-\Delta_{j}\right\rangle / \Delta_{j}$.

\section{Numerical simulation results}

\subsection{Validation of the plastic-damage model for concrete}

In order to verify and validate the performance of the proposed plastic-damage model for the concrete behavior, a single element of $10 \times 10 \mathrm{~cm}$ is modeled, as shown in Figure 1. This single element is modeled under the monotonic uniaxial compressive and monotonic uniaxial tensile loadings as well as the cyclic uniaxial tensile loading. The material parameters for the numerical modeling of the concrete element are obtained from the experimental test conducted by Gopalaratnam and Shah [32]. In Figure 2(a)

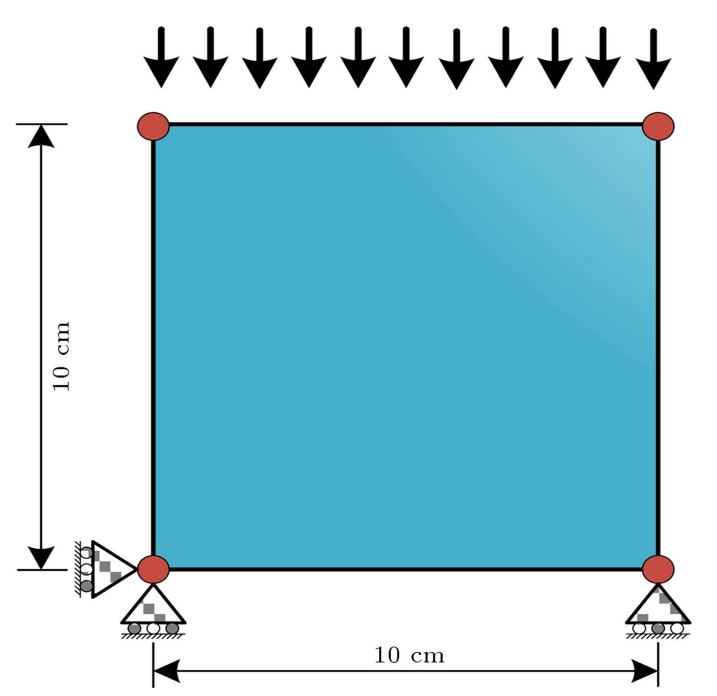

Figure 1. A concrete element proposed for verification of the plastic-damage model of concrete behavior.

and (b), the strain-stress curves are plotted for the monotonic uniaxial compression and uniaxial tension loadings. Complete agreement can be seen between the predicted results and the experimental stress-strain curves proposed for the compression and tension of the concrete. Also, the strain-stress curve of the tensile cyclic loading is plotted in Figure 2(c); it shows that the damage model can properly describe the behavior of concrete in tensile cyclic loading. It can be seen in Figure 2(c) that the strain-stress curve obtained by the numerical analysis is in excellent agreement with that obtained by the experimental test. This example clearly demonstrates that the proposed plastic-damage model can efficiently be used to capture the behavior of concrete.

\subsection{Validation of the cohesive fracture model for debonding}

In order to verify the cohesive interface model proposed between the FRP and concrete, the results of the model are compared with those of the debonding test reported by Au and Büyüköztürk [33]. The cohesive model is employed along the interface between the FRP and concrete through the FE analysis. The material properties of the concrete and FRP as well as the interface between them are obtained by the experimental test. In Figure 3, the setup of the experimental test is presented together with the interface fracture characterization of debonding in FRP plated concrete. In Figure 4, a comparison of the force-displacement curves is presented between the experimental and numerical results. It clearly shows that the cohesive interface model can properly capture the interface behavior between the FRP and concrete. The difference between two diagrams in the softening part of the force-displacement curve can be attributed 


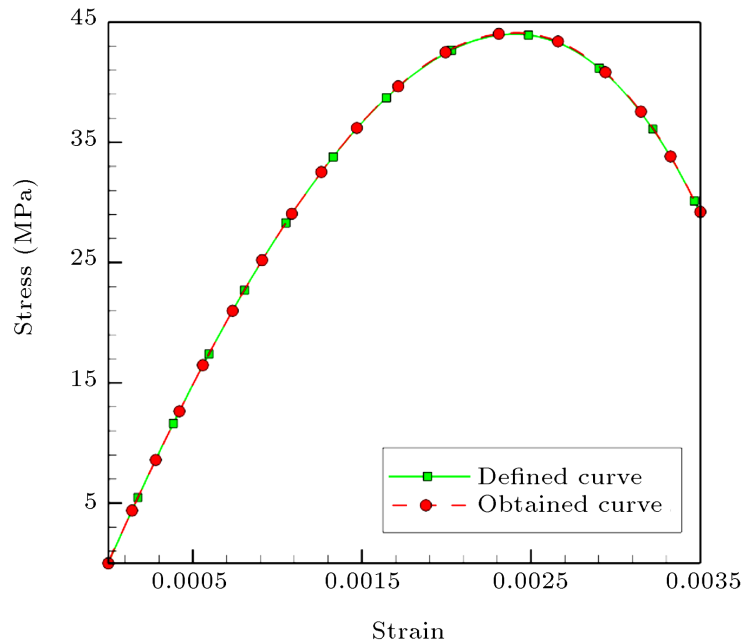

(a)

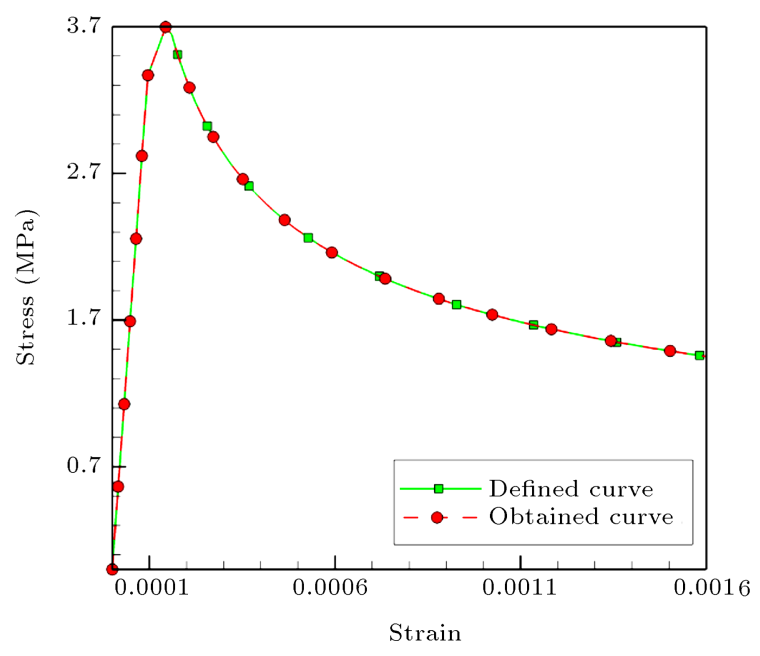

(b)

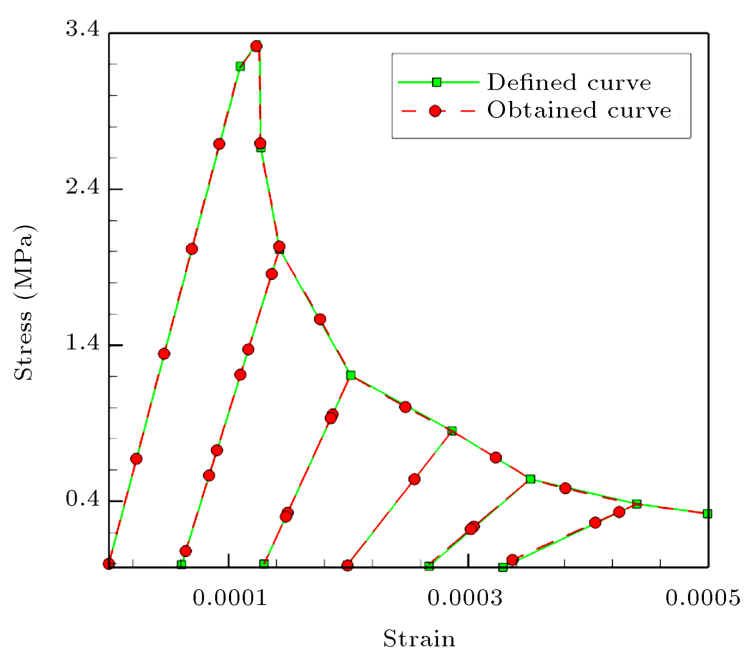

(c)

Figure 2. The stress-strain curves of a concrete element with the damage-plasticity model: (a) The monotonic uniaxial compression test, (b) the monotonic uniaxial tension test, and (c) the cyclic uniaxial tensile test.

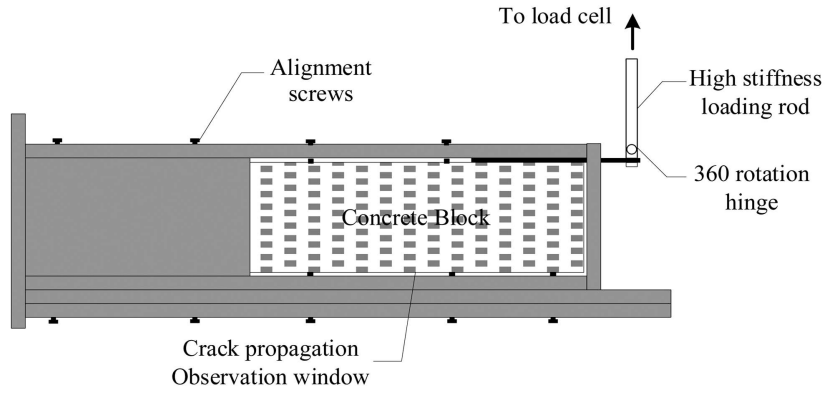

(a)

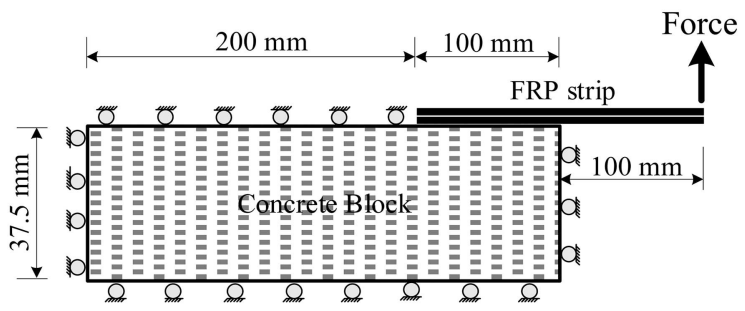

(b)

Figure 3. The debonding test: (a) The setup of experimental test and (b) the interface fracture of FRP strip together with the specimen configuration.

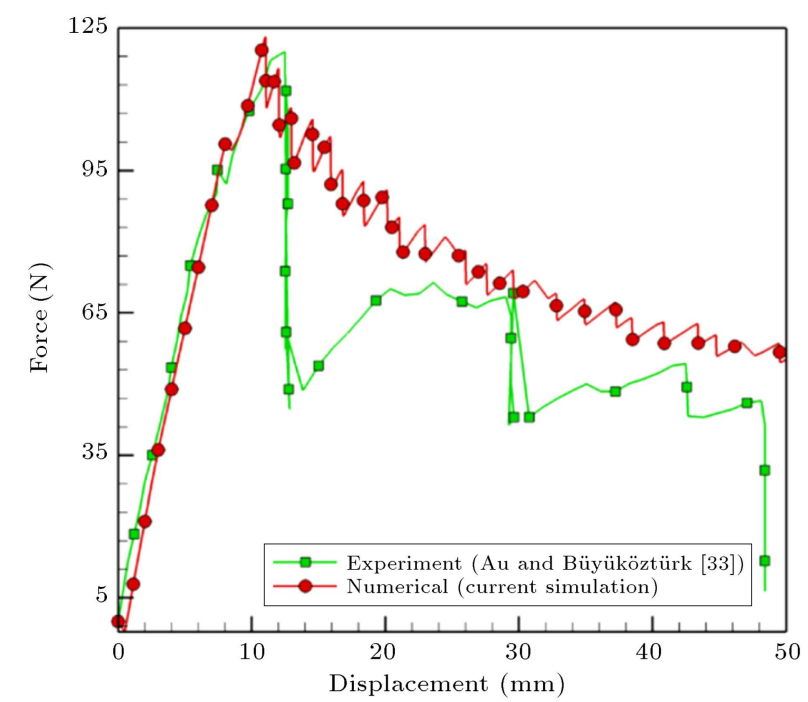

Figure 4. The debonding test; a comparison of the force-displacement curves between the experimental and numerical results.

to the lack of uniformity of the adhesive thickness and the presence of air bubbles.

\subsection{Numerical and experimental investigations into concrete arch}

In order to illustrate the performance of the proposed computational algorithm, the two desired concrete arches strengthened with FRP are analyzed numerically and the results are compared with the experimental tests; the first experiment was conducted by Zhang et al. [6] and the second one was conducted at the Strong Floor Laboratory of Sharif University 
of Technology [34]. In order to present the accuracy of the proposed computational algorithm, the forcedisplacement curves obtained by the finite element analysis are compared with those reported through the experimental tests. The reinforced concrete arch is modeled using the four-node bilinear element and the rebar is modeled using a two-node beam element. The rebars are connected to the concrete using the embedded technique inside the element.

In the first case, the concrete arch strengthened with internal FRP, originally conducted by Zhang et al. [6], is modeled using the proposed computational algorithm, as shown in Figure 5. In this figure, the concrete arch specimen is shown with its geometry, boundary conditions, and the FE mesh. It was observed in the experimental investigation that the cracks were first initiated in the middle of the span and then, propagated by increasing the load through the shoulders. It was also observed that a five-hinge arch was formed where the structure became unstable. It is interesting to highlight that the five-hinge structure was also detected through the numerical analysis of the concrete arch. In Figure 6, a com-

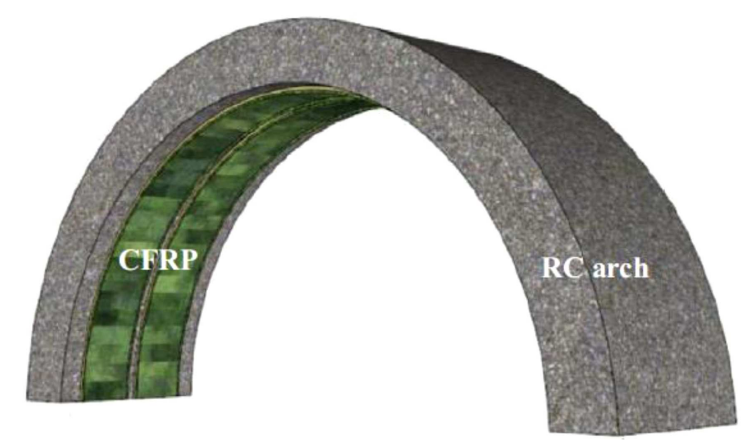

(a)

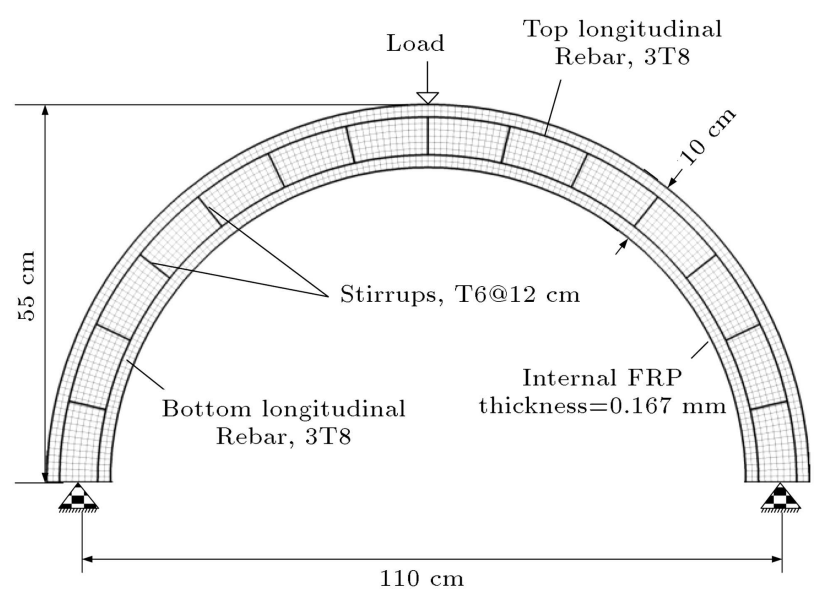

(b)

Figure 5. The concrete arch strengthened with internal FRP conducted by Zhang et al. [6]: (a) The concrete arch specimen and (b) the geometry, boundary conditions, and the FE mesh of the concrete arch.

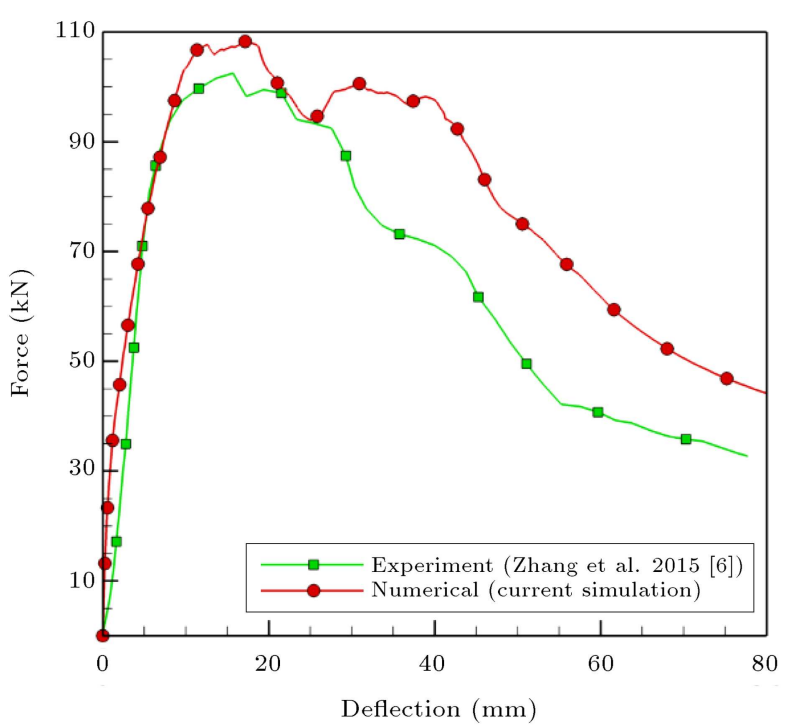

Figure 6. The concrete arch strengthened with internal FRP conducted by Zhang et al. [6]; a comparison of the force-displacement curves between the experimental and numerical results.

parison of the force-displacement curves is presented between the experimental and numerical results; it clearly demonstrates that the proposed computational algorithm can properly capture the behavior of the concrete arch. Obviously, a slight difference can be seen between the numerical analysis and experimental data at the beginning of the loading, which is due to the local splitting of the sample at the time of sitting of the sample; it cannot be modeled in the numerical model. Furthermore, the numerical analysis demonstrates higher strength than the experiment, which can be due to uncertainties in the properties of the materials utilized in the laboratory as well as the reduced concrete stress in high strains. According to the results, it can be concluded that the numerical analysis adequately shows good agreement with the experiments.

In the second case, the concrete arch strengthened with the internal and external FRPs experimentally conducted at the Strong Floor Laboratory of Sharif University of Technology [34] is modeled, as shown in Figure 7 . The data of one test is employed to verify the accuracy of the proposed computational model. The concrete arch specimen is $35 \mathrm{~cm}$ high with a span length of $110 \mathrm{~cm}$ and a rectangular cross-section with the dimensions of $30 \times 10 \mathrm{~cm}$. The internal and external parts of the arch are retrofitted by an FRP layer with the thickness of $0.35 \mathrm{~mm}$. Three longitudinal bars with a diameter of $8 \mathrm{~mm}$ and stirrups with a diameter of $6 \mathrm{~mm}$, with the spacing of $100 \mathrm{~mm}$, are used (Figure $7(\mathrm{c})$ ). All rebars have the strength of $400 \mathrm{MPa}$. The compressive strength of the concrete varies between 47 and $54 \mathrm{MPa}$; a compressive strength of $48 \mathrm{MPa}$ is considered in the numerical analysis. The 


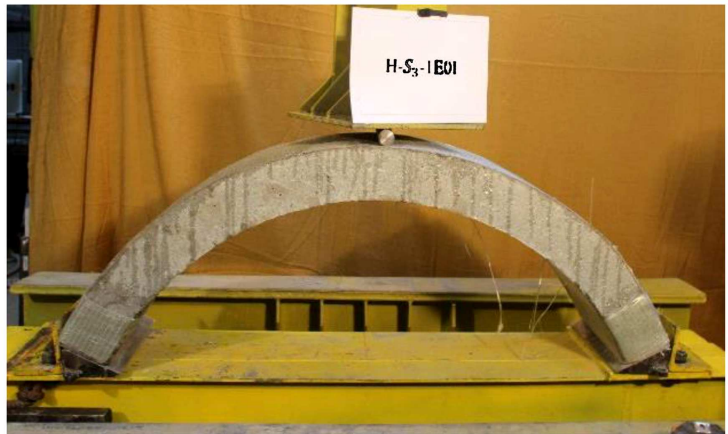

(a)

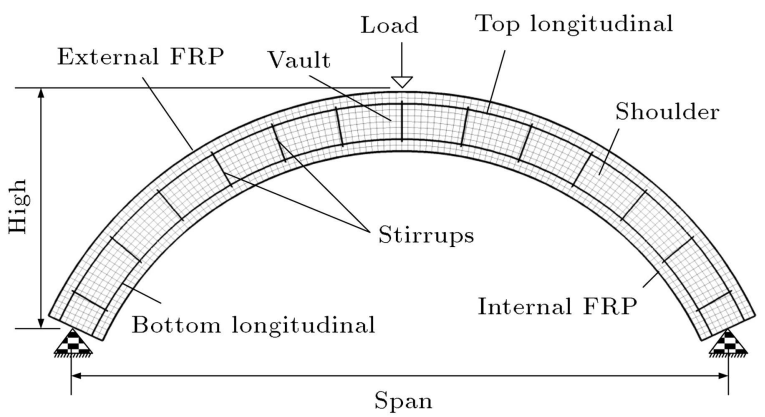

(b)

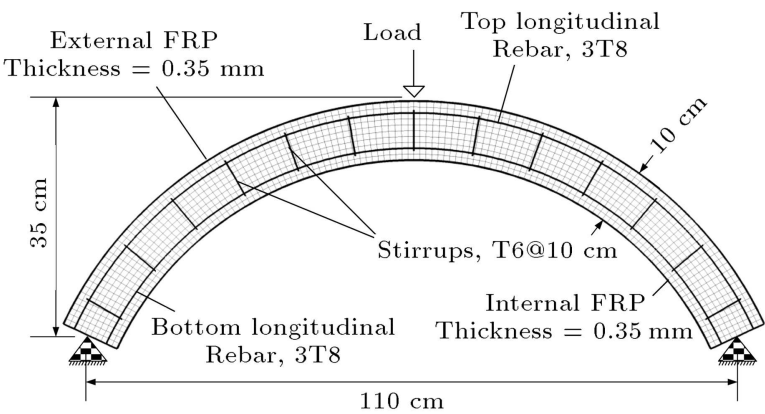

(c)

Figure 7. The concrete arch strengthened with internal and external FRPs conducted at Sharif University of Technology: (a) The setup of experimental test, (b) problem definition, and (c) the geometry, boundary conditions, and FE mesh of concrete arch.

strain-stress curves of the concrete in compression and tension are defined according to the recommendations of the CEB-FIP 2010. It can be observed that the failure of the arch occurs in a shear failure mode due to the excessive distance between the stirrups, as can be seen in Figure 8 . The maximum distance of the stirrups should be half the effective height of the section. Given the section height of $100 \mathrm{~mm}$, the stirrup spacing should be limited to a maximum of $50 \mathrm{~mm}$; however, the distance of stirrups is selected equal to $100 \mathrm{~mm}$ in the current experimental test for practical concerns. Because the tensile stress occurs along the interface between the FRP and concrete at the inner face of the arch, debonding happens at this region, as

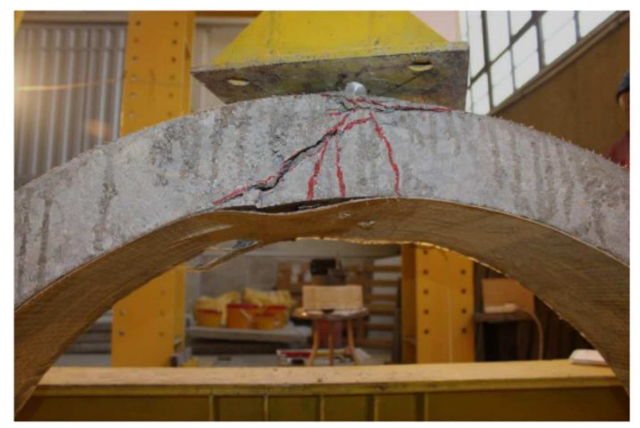

(a)

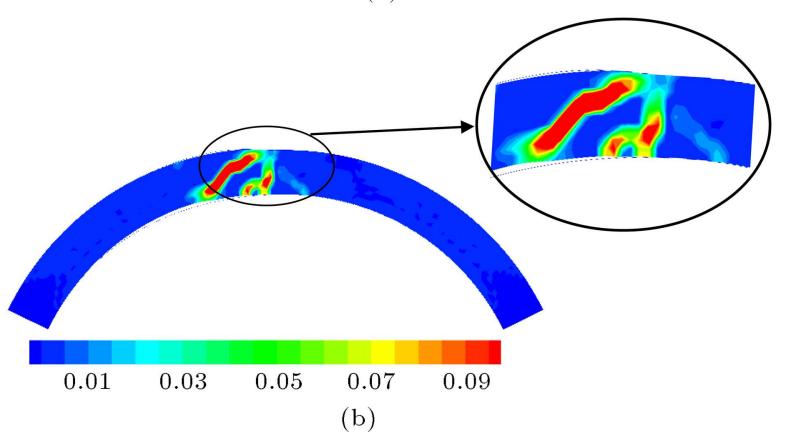

Figure 8. The concrete arch strengthened with internal and external FRPs conducted at Sharif University of Technology: (a) The crack trajectories obtained by experimental test and (b) the contour of plastic strain obtained by the numerical analysis.

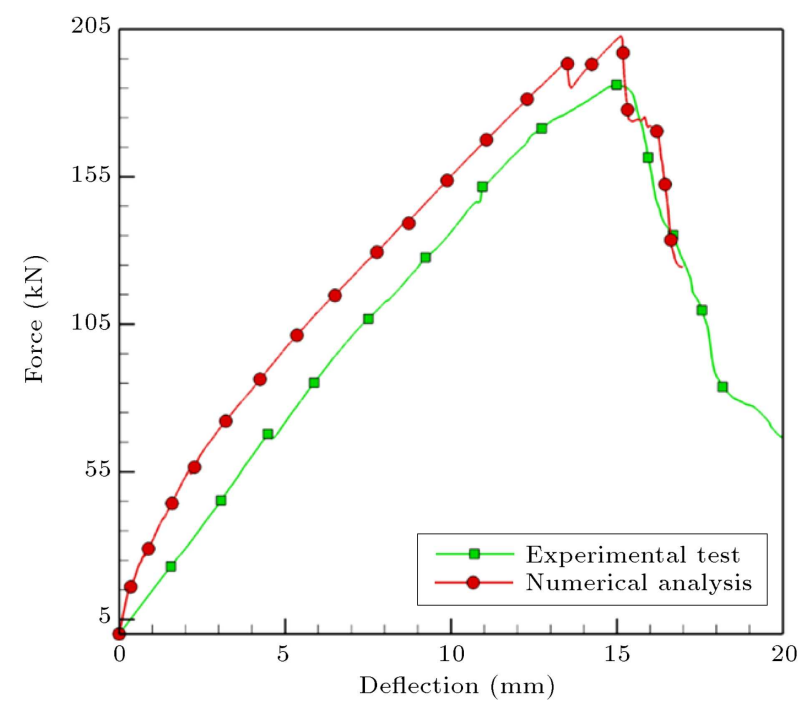

Figure 9. The concrete arch strengthened with internal and external FRPs conducted at Sharif University of Technology; a comparison of the force-displacement curves between the experimental and numerical results.

shown in Figure 8. A comparison between Figure 8(a) and (b) illustrates that the proposed computational algorithm can be used to accurately capture the crack trajectories. In Figure 9, the force-deflection curves are plotted for the experiment and numerical model, which are in good agreement. 


\section{Conclusion}

In the present paper, a computational technique is presented based on the plastic-damage model to investigate the effect of FRP strengthening on concrete arches. A plastic-damage model was utilized to capture the behavior of concrete. The interface between the FRP and concrete was modeled using a cohesive fracture model. In order to validate the accuracy of the concrete model, a single element was considered and exposed to monotonic tension, monotonic compression, and cyclic tension loads. Good agreement was observed between the predefined strain-stress curve and the strain-stress curve obtained by the numerical analysis. It demonstrated that the proposed plasticdamage model could efficiently be used to capture the behavior of concrete. Furthermore, the accuracy of the cohesive interface model between the FRP and concrete was investigated by comparing the results with the experimental data obtained by the debonding test. It clearly showed that the cohesive interface model could properly capture the interface behavior between the FRP and concrete. Finally, in order to illustrate the performance of the proposed computational algorithm, the two desired concrete arches strengthened with FRP were numerically analyzed and the results were compared with the experimental tests conducted at the Strong Floor Laboratory of Sharif University of Technology. It was shown that the results of the proposed computational model had adequate agreement with those of experiments.

\section{References}

1. Borri, A., Castori, G., and Corradi, M. "Intrados strengthening of brick masonry arches with composite materials", Composites Part B: Eng., 42, pp. 11641172 (2011).

2. Tao, Y., Stratford, T.J., and Chen, J.F. "Behaviour of a masonry arch bridge repaired using fibre-reinforced polymer composites", Eng. Struct., 33, pp. 1594-1606 (2011).

3. Chen, H., Zhou, J., Fan, H., et al. "Dynamic responses of buried arch structure subjected to subsurface localized impulsive loading: Experimental study", Int. J. Impact Eng., 65, pp. 89-101 (2014).

4. Hamed, E., Chang, Z.T., and Rabinovitch, O. "Strengthening of reinforced concrete arches with externally bonded composite materials: Testing and analysis", J. Composites Construc., 19, pp. 04014031 (2015).

5. Dagher, H.J., Bannon, D.J., Davids, W.G., et al. "Bending behavior of concrete-filled tubular FRP arches for bridge structures", Construc. Building Mater., 37, pp. 432-439 (2012).

6. Zhang, X., Wang, P., Jiang, M., et al. "CFRP strengthening reinforced concrete arches: Strength- ening methods and experimental studies", Composite Struct., 131, pp. 852-867 (2015).

7. Feenstra, P.H. and de Borst, R. "A composite plasticity model for concrete", Int. J. Solids Struct., 33, pp. 707-730 (1996)

8. Červenka, J. and Papanikolaou, V.K. "Three dimensional combined fracture-plastic material model for concrete", Int. J. Plasticity, 24, pp. 2192-2220 (2008).

9. Khoei, A.R. and Azami, A.R. "A single cone-cap plasticity with an isotropic hardening rule for powder materials", Int. J. Mech. Sciences, 47, pp. 94-109 (2005).

10. DorMohammadi, H. and Khoei, A.R. "A threeinvariant cap model with isotropic-kinematic hardening rule and associated plasticity for granular materials", Int. J. Solids Struct., 45, pp. 631-656 (2008).

11. Bažant, Z.P. and Ožbolt, J. "Nonlocal microplane model for fracture, damage, and size effect in structures", J. Eng. Mech., 116, pp. 2485-2505 (1990).

12. Voyiadjis, G.Z. and Abu-Lebdeh, T.M. "Damage model for concrete using bounding surface concept", J. Eng. Mech., 119, pp. 1865-1885 (1993).

13. Lubliner, J., Oliver, J., Oller, S., et al. "A plasticdamage model for concrete", Int. J. Solids Struct., 25, pp. 299-326 (1989).

14. Yazdani, S. and Schreyer, H.L. "Combined plasticity and damage mechanics model for plain concrete", $J$. Eng. Mech., 116, pp. 1435-1450 (1990).

15. Kattan, P.I. and Voyiadjis, G.Z. "A coupled theory of damage mechanics and finite strain elasto-plasticity - I. Damage and elastic deformations", Int. J. Eng. Science, 28, pp. 421-435 (1990).

16. Kattan, P.I. and Voyiadjis, G.Z. "A coupled theory of damage mechanics and finite strain elasto-plasticity II. Damage and finite strain plasticity", Int. J. Eng. Science, 28, pp. 505-524 (1990).

17. Lee, J. and Fenves, G.L. "Plastic-damage model for cyclic loading of concrete structures", J. Eng. Mech., 124, pp. 892-900 (1998).

18. Faria, R., Oliver, J., and Cervera, M. "A strain-based plastic viscous-damage model for massive concrete structures", Int. J. Solids Struct., 35, pp. 1533-1558 (1998).

19. Salari, M.R., Saeb, S., Willam, K.J., et al. "A coupled elastoplastic damage model for geomaterials", Comput. Meth. Applied Mech. Eng., 193, pp. 2625-2643 (2004).

20. Grassl, P. and Jirásek, M. "Damage-plastic model for concrete failure", Int. J. Solids Struct., 43, pp. 71667196 (2006).

21. Nguyen, G.D. and Korsunsky, A.M. "Damageplasticity modelling of concrete: calibration of parameters using separation of fracture energy", Int. J. Fracture, 139, pp. 325-332 (2006). 
22. Nguyen, G.D. and Houlsby, G.T. "A coupled damageplasticity model for concrete based on thermodynamic principles: Part I: model formulation and parameter identification", Int. J. Numer. Analy. Meth. Geomech., 32, pp. 353-389 (2008).

23. Nguyen, G.D. and Houlsby, G.T. "A coupled damageplasticity model for concrete based on thermodynamic principles: Part II: non-local regularization and numerical implementation", Int. J. Numer. Analy. Meth. Geomech., 32, pp. 391-413 (2008).

24. Moslemi, H. and Khoei, A.R. "3D modeling of damage growth and crack initiation using adaptive finite element technique", Scientia Iranica, Trans. A., J. Civil Eng., 17, pp. 372-386 (2010).

25. Khoei, A.R., Eghbalian, M., Azadi, H., et al. "Numerical simulation of ductile crack growth under cyclic and dynamic loading with a damage-viscoplasticity model", Eng. Fracture Mech., 99, pp. 169-190 (2013).

26. Broumand, P. and Khoei, A.R. "The extended finite element method for large deformation ductile fracture problems with a non-local damage-plasticity model", Eng. Fracture Mech., 112, pp. 97-125 (2013).

27. Broumand, P. and Khoei, A.R. "X-FEM modeling of dynamic ductile fracture problems with a nonlocal damage-viscoplasticity model", Finite Elements Anal. Design, 99, pp. 49-67 (2015).

28. Khoei, A.R., Extended Finite Element Method: Theory and Applications, John Wiley (2015).

29. Khoei, A.R., Moslemi, H., Ardakany, K.M., et al. "Modeling of cohesive crack growth using an adaptive mesh refinement via the modified-SPR technique", Int. J. Fracture, 159, pp. 21-41 (2009).

30. Khoei, A.R., Moslemi, H., and Sharifi, M. "Threedimensional cohesive fracture modeling of non-planar crack growth using adaptive $\mathrm{FE}$ technique", Int. J. Solids Struct., 49, pp. 2334-2348 (2012).

31. Turon, A., Camanho, P.P., Costa, J., et al. "A damage model for the simulation of delamination in advanced composites under variable-mode loading", Mech. Mater., 38, pp. 1072-1089 (2006).

32. Gopalaratnam, V. and Shah, S.P. "Softening response of plain concrete in direct tension", ACI Mater. J., 82, pp. 310-323 (1985).

33. Au, C. and Büyüköztürk, O. "Peel and shear fracture characterization of debonding in FRP plated concrete affected by moisture", J. Composites Construc., 10, pp. 35-47 (2006).

34. Moradi, H., Khaloo, A., Shekarchi, M., and Kazemian, A. "Effect of glass fiber-reinforced polymer on flexural strengthening of RC arches", Scientia Iranica, Transactions A, 26(4), pp. 2299-2309 (2019).

\section{Biographies}

Tahmaz Ahmadpour received his BSc degree in Civil Engineering from Iran University of Science and Technology, Tehran, Iran, in 2001 and his MSc degree in Structural Engineering from Sahand University of Technology, Tabriz, Iran, in 2004. He is currently a $\mathrm{PhD}$ candidate in Civil Engineering at the International Campus of Sharif University of Technology. His main research interests are computational fracture and damage mechanics for brittle materials and Extended Finite Element Method (X-FEM).

Yousef Navid Tehrani received his BSc degree in Civil Engineering from Iran University of Science and Technology, Tehran, Iran, in 2014 and his MSc degree in Structural Engineering from Sharif University of Technology, Tehran, Iran, in 2017. He is currently working as a Research Assistant with Professor Amir R. Khoei. His main research interests are computational plasticity, computational fracture and damage mechanics, material characterization, and multiscale modeling.

Amir R. Khoei received his $\mathrm{PhD}$ in Civil Engineering from the University of Wales Swansea in UK in 1998. He is currently a Professor in the Civil Engineering Department at Sharif University of Technology. He is a member of the editorial board in the journals of Finite Elements in Analysis and Design and European Journal of Computational Mechanics. He has been selected several times as a Distinguished Professor at Sharif University of Technology and was selected as a Distinguished Professor by the Ministry of Science, Research and Technology in 2008. He is the silver medal winner of Khwarizmi International Award organized by the Iranian Research Organization for Science and Technology. 